\title{
Cylindrical Resonator Technique for Ultrasonic Absorption Measurement and Its Application to Cellulose Acetate-Cyclohexanone System
}

\author{
Shigeo KATo, Hiroyasu NOMURA, and Yutaka MIYAHARA \\ Department of Chemical Engineering, Faculty of Engineering, \\ Nagoya University, Chikusa-ku, Nagoya 464, Japan.
}

(Received September 5, 1978)

\begin{abstract}
Measurements of ultrasonic absorption were carried out for cellulose acetate in cyclohexanone at frequencies from 0.5 to $130 \mathrm{MHz}$ over a temperature range of 0 to $30^{\circ} \mathrm{C}$. The molecular weight of the sample was $5.8 \times 10^{4}$. The apparatus was set up according to the cylindrical resonator technique to determine ultrasonic absorption coefficients with high accuracy on the low frequency side, especially below $10 \mathrm{MHz}$. The relaxation process observed could not be expressed by discrete relaxation mechanisms, such as single or double relaxations. This relaxation process may be expressed well by an equation which has a broad distribution of relaxation frequencies, such as the following,

$$
\frac{\alpha}{f^{2}}=\int_{\tau_{\alpha}}^{\tau_{\beta}} \frac{F(\tau)}{1+\omega^{2} \tau^{2}} \mathrm{~d} \tau+B, \quad F(\tau)=\frac{A \tau_{\alpha}^{1 / 2}}{\left(\tau-\tau_{\alpha}\right)^{1 / 2}}
$$

In the cellulose acetate cyclohexanone system, the segmental motions of the polymer chain are strongly coupled with various molecular motions, such as normal mode motion (Rouse-Zimm type) and intramolecular motion.

KEY WORDS Polymer Solution / Cellulose Acetate / Ultrasonic Relaxation / Cylindrical Resonator Technique / Segmental Motion /
\end{abstract}

In our previous papers, ${ }^{1-2}$ we reported that ultrasonic relaxation processes were observed in solutions of flexible, vinyl type, and semiflexible polymers in the $\mathrm{MHz}$ region. To get further infomation on chain dynamics in solution, studies on stiff polymers, such as cellulose and its derivatives, are inevitably needed. For this purpose, very accurate measurements should be carried out on the low-frequency side, below $10 \mathrm{MHz}$.

Several techniques such as reverberation, interferometer, and pulse techniques have been used to measure ultrasonic absorption below $10 \mathrm{MHz}$. However, these techniques have some disadvantages, such as the need for large quantities of sample and sophisticated experimental techniques and troublesome correction for diffraction effect of sound.

Recently, ${ }^{3}$ Eggers presented a new technique utilizing a cylindrical resonator. This technique requires only small quantities of sample and most of the disadvantages experienced in the earlier techniques have been done away with.

Our apparatus measures ultrasonic absorption in a frequency range from 0.5 to $10 \mathrm{MHz}$, using this technique. In this paper, we report on the experimental conditions for making correct measurements and the results of the ultrasonic measurements of cellulose acetate in cyclohexanone.

\section{PRINCIPLE OF THE RESONATOR TECHNIQUE}

Figure 1(a) shows a block diagram of the cylindrical resonator technique. Our apparatus consists simply of a signal generator, a resonator cell, and detectors. The cell consists of a liquid column and two quartz transducers. A tunable sine wave oscillator drives the input quartz transducer and pro- 


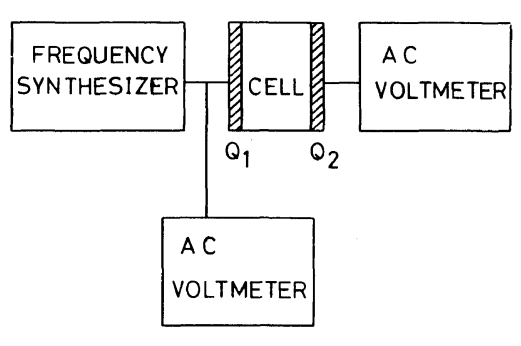

(a)

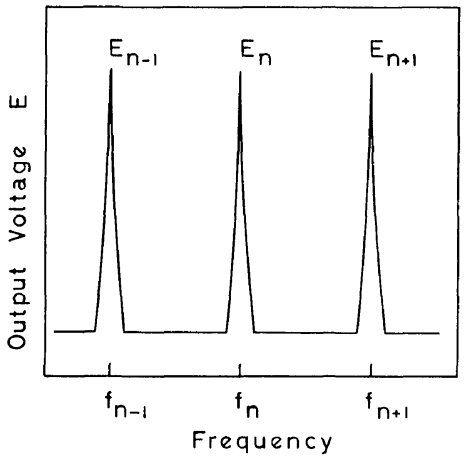

(b)

Figure 1. (a) Block diagram of the cylindrical-resonator technique. (b) Resonance peaks and frequencies.

duces a standing wave in the liquid column at characteristic frequencies $f_{n}$. At these frequencies, the output quartz delivers pronounced voltage peaks shown schematically in Figure 1(b). If the ultrasonic absorption measurement could be performed with an "ideal" resonator containing only the energy loss due to the liquid, then the ultrasonic absorption per wavelength $\alpha \lambda$ in the liquid would be given by

$$
\frac{\alpha \lambda}{\pi}=\frac{\Delta f_{n}}{f_{n}}=\frac{1}{Q}
$$

where $\Delta f_{n}$ and $f_{n}, \alpha$ and $\lambda$ are half power band widths (HPB) of a resonance peak and its frequency, the absorption coefficient in the liquid and the wavelength, respectively. $Q$ is the quality factor of the resonator. Actually, the "real" resonator contains energy losses of various types other than that of the liquid, due to the beam divergence, friction effect between liquid and side wall of the resonator, scattering, and imperfect reflection at the quartz surface, etc.

Therefore, energy loss of the "real" resonator $(1 / Q)_{\mathrm{R}}$ is given by

$$
\left(\frac{1}{Q}\right)_{\mathrm{R}}=\left(\frac{1}{Q}\right)_{\mathrm{L}}+\left(\frac{1}{Q}\right)_{\mathrm{M}}
$$

where $(1 / Q)_{\mathrm{L}}$ is the loss due to sound attenuation in the liquid and $(1 / Q)_{\mathrm{M}}$ are the other contributions. Elimination of $(1 / Q)_{\mathrm{M}}$ can be done by a reference measurement in the same cell at the same frequencies with a suitable liquid. Then the excess absorption per wavelength $(\alpha \lambda)_{\text {excess }}$ in the liquid is obtained from eq 3 .

$$
(\alpha \lambda)_{\mathrm{excess}}=\pi\left[\left(\Delta f_{\mathrm{s}}-\Delta f_{\mathrm{r}}\right) / f_{n}\right]
$$

In eq $3, \Delta f_{\mathrm{s}}$ and $\Delta f_{\mathrm{r}}$ represent the corresponding HPB values of the $n$-th resonance peak in the sample and reference liquid, respectively.

\section{TESTS FOR THE APPARATUS}

The cylindrical-resonator technique requires a signal generator with high stability and high resolution of frequency and with low harmonic distortion. Therefore, two frequency synthesizers were used as the signal generator. One was the Rockland Model $5100(0-2 \mathrm{MHz})$ and the other was the John-Fluke Model 6039 A (above $2 \mathrm{MHz}$ ). As detectors, Y.H.P. Model 3400 A RMS voltmeters were used. Three cylindrical resonator cells were used for different frequency ranges. Table I and Figure 2 show the details of these cells. To obtain a high $Q$ value for the resonator, the fundamental frequencies of two transducers were matched within $0.005 \%$ and their surfaces were polished within optical grade. The temperature drift in the cell causes a drift of resonance frequency and this makes absorption measurement inaccurate. The temperature in the cell was controlled within $\pm 0.01^{\circ} \mathrm{C}$.

Three kinds of tests were carried out to determine whether our apparatus is actually reliable. In the first step, resonance frequencies observed were compared with those calculated from mathematical models. At this stage, three types of models for the resonator cell have been proposed by Eggers, ${ }^{3}$ Dev, et al.,${ }^{4}$ and Labhardt, et al., ${ }^{5}$ respectively. Though these models do not perfectly represent the "real" resonator which 
Cylindrical Resonator Technique for Ultrasonic Absorption Measurement

Table I. Details of cells

\begin{tabular}{crrlcccc}
\hline Cell & \multicolumn{3}{c}{ Size, cm } & $\begin{array}{c}\text { Effective volume, } \\
\mathrm{m} l\end{array}$ & $\begin{array}{c}\text { Effective diameter } \\
\text { of quartz, cm }\end{array}$ & $\begin{array}{c}\text { Fundamental } \\
\text { frequency, MHz }\end{array}$ \\
\cline { 2 - 4 } & $\mathrm{L}$ & $\mathrm{W}$ & $\mathrm{H}$ & & 13 & 3.0 & 2 \\
$\mathrm{~A}$ & 10 & 20 & 11 & 15 & 55 & 5.0 & 2 \\
$\mathrm{~B}$ & 18 & 17 & 15.5 & 4 & 2.6 & 5 \\
$\mathrm{C}$ & 8 & 12 & 11 & & 45 & \\
\hline
\end{tabular}

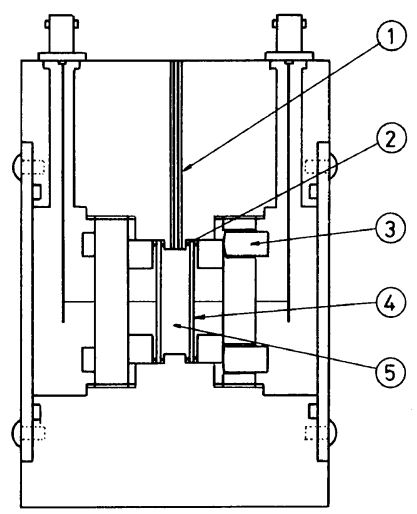

Figure 2. Cylindrical resonator cell: 1, filling hole; 2, Oring; 3, screw; 4, quartz transducer; 5, cavity.

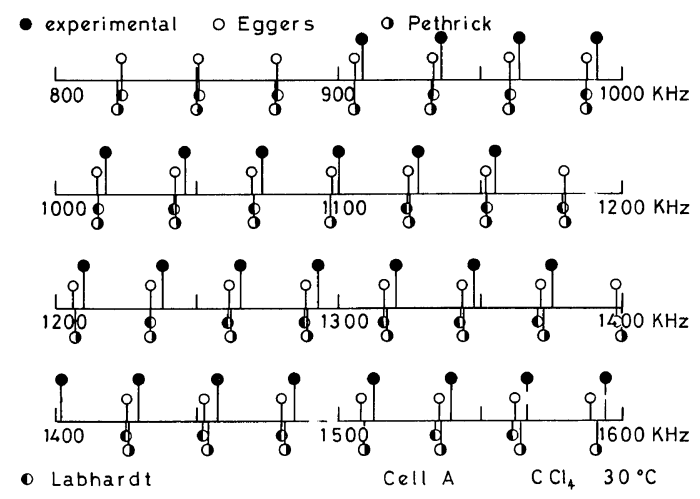

Figure 3. Comparison of observed resonance frequencies with theoretical values: $\bigcirc$, experimental; $\bigcirc$, Eggers; o, Pethrick; D, Labhardt.

contains the many losses mentioned above, they give fairly good estimations of resonance frequencies. As is seen in Figure 3, the observed frequencies are in good agreement with those calculated. In the "real" resonator, unestimated vibrational modes of higher order are observed. The coupling of the principal mode with these modes causes broadening effects in the resonance peak: this makes velocity determi- nations inaccurate and absorption measurements meaningless. Therefore, a careful selection of the principal mode is needed to determine the absorption coefficient accurately. The second test was carried out by measurements on non-relaxing liquids.

Equation 1 can be written as

$$
\Delta f_{n}=\frac{\alpha}{f_{n}^{2}} \cdot \frac{v}{\pi} \cdot f_{n}^{2}
$$

where $v$ is the velocity of the sound in the liquid. In the non-relaxing liquids, $\alpha / f_{n}{ }^{2} \cdot v / \pi$ is independent of the frequency; therefore, $\Delta f_{n}$ should vary linearly with the square of the resonance frequency, $f_{n}^{2}$. Figure 4 shows the relationships between $f_{n}$ and $\Delta f_{n}$ in water. As may be expected from eq 4, the plot of $\log \left(\Delta f_{n}\right) v s \cdot \log \left(f_{n}\right)$ shows a straight line with a slope of 2. Moreover, the observed values of $\Delta f_{n}$ are in good agreement with those calculated from the values previously reported. This fact indicates that cells act practically as "ideal" resonators. The third and most severe test for our apparatus was to examine whether eq 2 is satisfied or not in our cell. Using the observed values of $(1 / Q)_{\mathrm{R}}$ and eq 2 , mechanical losses $(1 / Q)_{\mathrm{M}}$ were estimated in benzene and toluene, independently. They should agree with one another, for benzene and toluene have similar acoustic impedances. As the value of $(1 / Q)_{\mathrm{L}}$ in benzene is very different from that of toluene, a strict test is possible. Values in the literature were used as $(1 / Q)_{\mathrm{L}}$. As seen in Figure 5, the calculated values of $(1 / Q)_{\mathrm{M}}$ are in good agreement with one another. The difference $\Delta \alpha / f^{2}$ of the absorption coefficients between benzene and toluene can be calculated from corresponding HPB values, using eq 3. Figure 6 shows the relationship between $\Delta \alpha / f^{2}$ and $f_{n}$. Taking into account the standard deviation of the values in the literature, the observed values should exist between two chain lines in Figure 6. The observed values fall exactly within this limit.

The results of the three tests mentioned above 


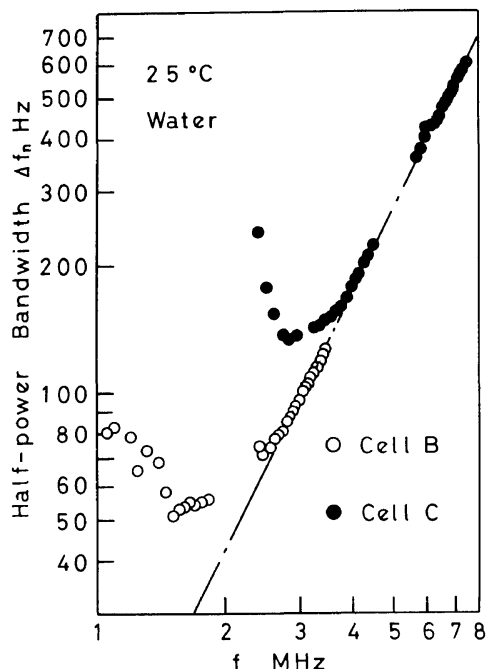

Figure 4. Relationship between $f_{n}$ and $\Delta f_{n}$ : at $25^{\circ} \mathrm{C}$ in water: $\bigcirc$, cell $\mathrm{B}$; $\boldsymbol{O}$, cell $\mathrm{C}$; ---, $\alpha v / \pi$ for water.

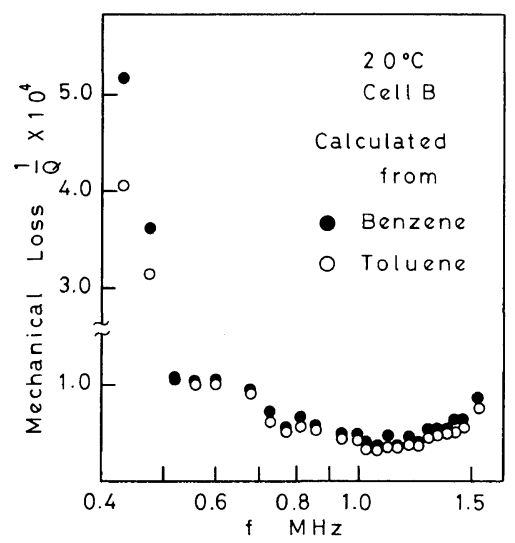

Figure 5. Estimation of mechanical losses $(1 / Q)_{\mathrm{M}}$ from benzene and toluene: $\bigcirc$, benzene; $\bigcirc$, toluene.

clearly indicate that our apparatus can provide reliable results.

\section{ULTRASONIC ABSORPTION MEASUREMENT \\ ON CELLULOSE ACETATE IN CYCLOHEXANONE}

The cellulose acetate, abbreviated as CA hereafter, (acetyl $39.4-\%$ ASTM viscosity 45 ) was supplied by Eastman Kodak Co. Ltd. and its molecular weight was $5.8 \times 10^{4}$. Cyclohexanone, abbreviated as $\mathrm{CHN}$ hereafter, was purified by distillation after dehy-

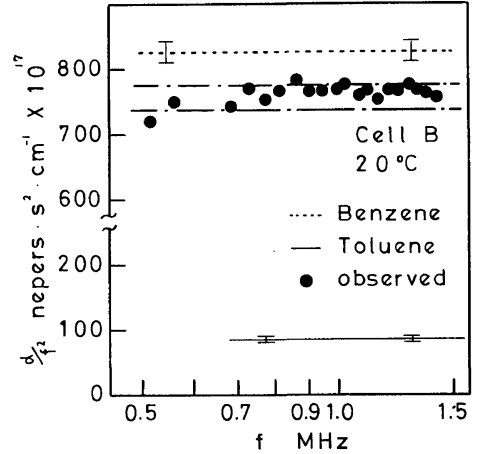

Figure 6. Difference in ultrasonic-absorption coefficients between benzene and toluene (mean literature values): ---, benzene; - , toluene; I, deviation; observed.

dration. The concentration of the solution measured was $3.56 \mathrm{~g} / 100 \mathrm{ml}$. An ultrasonic absorption measurement was carried out using the resonator technique and pulse technique. The frequency range measured was from 0.5 to $130 \mathrm{MHz}$ and temperature range was from 0 to $30^{\circ} \mathrm{C}$. Density, viscosity, and sound velocity of the solution were determined by methods reported previously. ${ }^{1}$

Ultrasonic relaxation phenomena of $\mathrm{CHN}$ could not be observed in this experiment. Figure 7 shows the ultrasonic absorption coefficients of the solution plotted as a function of frequency. Usually, in solutions of vinyl-type polymers, discrete-ultrasonicrelaxation processes have been observed in the $\mathrm{MHz}$ region and have been attributed to local segmental motion $^{2,6}$ of the polymer chain. As is apparent from Figure 7 , the relaxation process observed in this

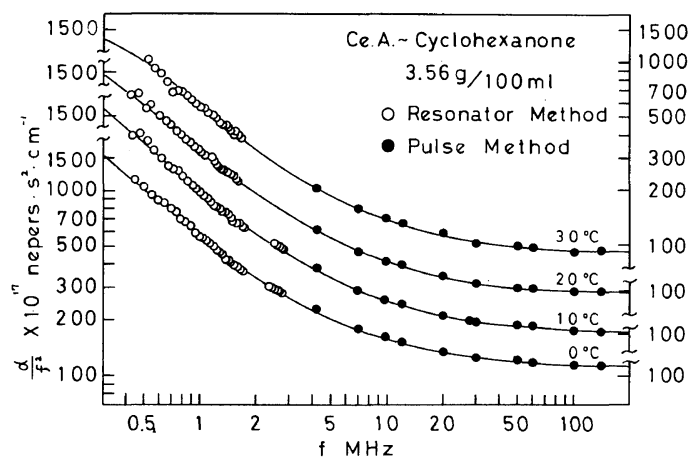

Figure 7. Frequency dependence of ultrasonicabsorption of cellulose acetate in cyclohexanone: $\bigcirc$, resonator method; $\bigcirc$, pulse method. 
solution could not be expressed by discreterelaxation mechanisms, such as single or double relaxations.

Therefore, the multi-relaxation processes with a broad distribution of relaxation frequencies should be considered. One possible way to express the multirelaxation processes, as proposed by Pethrick, et al., ${ }^{6}$ is to apply the Cole-Cole type equation to the ultrasonic relaxation processes. However, as pointed out by Saito, ${ }^{7}$ the parameter $\beta$ in the Cole-Cole type equation has only a indirect physical meaning. Thus, it is better to discuss the relaxation process using a relaxation equation directly containing the relaxation spectrum. Grigorev, et al.,$^{8}$ have proposed such an equation with two types of relaxation spectrums. However, their equation cannot express the present experimental results. As is seen in Figure 7 , it is possible to analyze our data with an equation having one relaxation spectrum.

We adopt the following equation similar to these.

$$
\frac{\alpha}{f^{2}}=\int_{\tau_{\alpha}}^{\tau_{\beta}} \frac{F(\tau)}{1+\omega^{2} \tau^{2}} \mathrm{~d} \tau+B
$$

where $\tau_{\alpha}, \tau_{\beta}$, and $\omega$ are the minimum and maximum relaxation times in the relaxation spectrum and the angular frequency, respectively. Parameter $B$ represents the absorptions due to other ultrasonicabsorption mechanisms. For a distribution function of relaxation times, $F(\tau)$, we assume the following wedge-type equation from the analogy to the relaxation spectrum of dynamic shear moduli in polymer solutions,

$$
F(\tau)=\frac{A \tau_{\alpha}{ }^{1 / 2}}{\left(\tau-\tau_{\alpha}\right)^{1 / 2}}
$$

where $A$ is the parameter in the distribution function of the relaxation strength $r(\tau)$. Assuming the following equations,

$$
\begin{aligned}
& r(\tau)=0, \quad \tau<\tau_{\alpha}, \tau>\tau_{\beta} \\
& r(\tau)=\frac{A v \tau_{\alpha}{ }^{1 / 2}}{2 \pi^{2} \tau\left(\tau-\tau_{\alpha}\right)^{1 / 2}}, \quad \tau_{\alpha} \leqq \tau \leqq \tau_{\beta}
\end{aligned}
$$

we can write $F(\tau)$ as the form of eq 6 (see APPENDIX). According to Gotlib, et al., ${ }^{9}$ the parameter $A$ can be written in terms of the number of segments and the density of the solution, etc. In Figure 7, the solid lines represent the best fit, using eq 5 and 6 . Parameters in eq 5 and 6 are summarized in Table II.
As is apparent in Table II, the ultrasonic relaxation process of $\mathrm{CA}$ in $\mathrm{CHN}$ can be explained by considering the very broad distribution of relaxation times over about three decades. The very high values of $f_{\alpha}$ obtained are not so unreasonable, for vibrational relaxation in the normal organic liquids is usually observed in $\mathrm{GHz}$ region. ${ }^{10}$ The values of $f_{\beta}$ are in several hundreds of $\mathrm{KHz}$.

In the $\mathrm{KHz}$ region, dielectric relaxation processes have been observed in dilute solutions of amylose acetate and ethyl cellulose by Imamura and his coworkers. ${ }^{11}$ These relaxation processes depend on molecular weight and are thus attributed to the rotation of the molecules as a whole. As a very crude approximation, we have project their results to our experiment, and found that the relaxation process due to rotation exists in a frequency range from $10^{4}$ to $10^{5} \mathrm{~Hz}$. Therefore, there are some possibilities that this mode is coupled with the segmental motions in the polymer chain on higher-frequency side.

On the other hand, Wang and $\mathrm{Zimm}^{12}$ recently modified the bead-spring model theories developed by Rouse ${ }^{13}$ and $\mathrm{Zimm}^{14}$, taking into account the effects of interactions among chain molecules. In a moderately concentrated polymer solution, it is more practical to estimate the relaxation times on the basis of Wang-Zimm theory rather than the original Rouse and Zimm theories. According to Wang and Zimm, the relaxation times, $\tau_{k}$, of normal mode motion are given by the following equation,

$$
\tau_{k}=\frac{M \eta \eta_{\mathrm{sp}}(0)}{N_{\mathrm{A}} k T c \lambda_{k}\left(\sum_{k=1}^{N} \frac{1}{\lambda_{k}}\right)}
$$

where $\eta_{\mathrm{sp}}(0)$ is the specific viscosity at steady state and $k$ and $T$ are the Boltzmann constant and the absolute temperature, respectively. In eq $8, \tau_{k}$ are eigenvalues, $\eta$ is the viscosity of solvent, $N$ is the number of springs, $M$ is the molecular weight of polymer, $N_{\mathrm{A}}$ is Avogadro's number, and $c$ is the concentration of the polymer in mass per unit volume. Using the experimental values of steadystate viscosity and eq 8 , we can estimate the relaxation times which should be measurable in the several-hundreds- $\mathrm{KHz}$ region. These estimated relaxation times are follows.

$$
\begin{aligned}
& \tau_{9}=6.9 \times 10^{-7} \mathrm{~s}, \tau_{10}=5.7 \times 10^{-7} \mathrm{~s}, \\
& \tau_{11}=4.8 \times 10^{-7} \mathrm{~s}, \text { etc. }
\end{aligned}
$$

Therefore, it seems plausible that the normal mode 
Table II. Relaxation parameters for $\mathrm{CA}$ in $\mathrm{CHN}$

\begin{tabular}{rcccccc}
\hline, & $\begin{array}{c}A \times 10^{7}, \\
\mathrm{~s} / \mathrm{cm}\end{array}$ & $\begin{array}{c}B \times 10^{7}, \\
\mathrm{~s}^{2} / \mathrm{cm}\end{array}$ & $\begin{array}{c}\tau_{\beta} \times 10^{7}, \\
\mathrm{~s}\end{array}$ & $\begin{array}{c}\tau_{\alpha} \times 10^{10}, \\
\mathrm{~s}\end{array}$ & $\begin{array}{c}f_{\beta}, \\
\mathrm{MHz}\end{array}$ & $\begin{array}{c}f_{\alpha}, \\
\mathrm{MHz}\end{array}$ \\
\hline 30 & 5.61 & 91 & 4.0 & 3.2 & 0.4 & 500 \\
20 & 6.75 & 96 & 5.3 & 2.7 & 0.3 & 600 \\
10 & 7.57 & 102 & 8.0 & 3.2 & 0.2 & 500 \\
0 & 7.29 & 112 & 8.0 & 3.2 & 0.2 & 500 \\
\hline
\end{tabular}

motions of a higher order strongly effect the ultrasonic-relaxation process in the $\mathrm{MHz}$ region.

The other possible mechanism is that proposed by Tobolsky, et al. ${ }^{15}$ who say that the damped torsional oscillation in a polymer chain also gives rise to the same relaxation spectrum as eq 6 and the dispersion region related to this mechanism exists on a frequency side higher than that related to Rouse-Zimm model. Considering the molecular structure and the stiffness of the CA chain, it is also possible to explain the present result using the model of Tobolsky, et al.

In spite of our efforts to measure the ultrasonic absorption on the lower-frequency side, it is difficult, at this stage, to conclude which mechanism contributes mainly to the relaxation process of $\mathrm{CA}$ in $\mathrm{MHz}$ region. However, we can say, at least, that in the case of $\mathrm{CA}-\mathrm{CHN}$ system, the segmental motions of polymer chain are coupled with various molecular motions, such as the normal mode motion of the Rouse-Zimm type and intramolecular motions over a very-wide-frequency range.

\section{APPENDIX}

In general, a multi-stage relaxation equation of ultrasonic absorption can be written by

$$
\frac{\alpha}{f} \frac{v_{0}^{2}}{v}=\pi \sum_{j=1}^{n} \frac{r_{j} \omega \tau_{j}}{1+\omega^{2} \tau_{j}^{2}}
$$

where $v_{0}$ is the sound velocity at the limit of zero frequency, $r_{j}$ is the relaxation strength of the $j$-th relaxation step. If the relaxation times distribute very closely to one another, one can replace the summations in eq (A-1) by integral. Let $r(\tau) \mathrm{d} \tau$ be the contribution to the relaxation strength from a process whose relaxation times distribute between $\tau$ and $\tau+\mathrm{d} \tau$; then eq (A-1) may be written in the form

$$
\frac{\alpha v_{0}^{2}}{f \quad v}=\pi \int_{0}^{\infty} \frac{r(\tau) \omega \tau}{1+\omega^{2} \tau^{2}} \mathrm{~d} \tau
$$

Equation (A-2) can be arranged as follows.

$$
\frac{\alpha}{f^{2}} \frac{v_{0}^{2}}{v}=\pi \int_{0}^{\infty} \frac{2 \pi r(\tau) \tau}{1+\omega^{2} \tau^{2}} \mathrm{~d} \tau
$$

If the dispersion of sound velocity is very small, $v_{0}$ may be considered as $v$. Therefore, the following equation is obtained,

$$
\frac{\alpha}{f^{2}}=\int_{0}^{\infty} \frac{2 \pi^{2} r(\tau) \tau}{v\left(1+\omega^{2} \tau^{2}\right)} \mathrm{d} \tau
$$

In the case of a single relaxation process, this equation is reduced to the familiar one as follows.

$$
\frac{\alpha}{f^{2}}=\frac{2 \pi^{2} \cdot r \tau}{v\left(1+\omega^{2} \tau^{2}\right)}+B
$$

Comparing eq (A-4) with eq 6 , we get the following equation,

$$
\begin{aligned}
& r(\tau)=0, \quad \tau<\tau_{\alpha}, \tau>\tau_{\beta} \\
& r(\tau)=\frac{A v \tau_{\alpha}^{1 / 2}}{2 \pi^{2} \tau\left(\tau-\tau_{\alpha}\right)^{1 / 2}}, \quad \tau_{\alpha} \leqq \tau \leqq \tau_{\beta}
\end{aligned}
$$

\section{REFERENCES}

1. S. Kato, I. Uehara, H. Kondo, H. Nomura, and Y. Miyahara, Nippon Kagaku Kaishi, 1651 (1975).

2. S. Kato, H. Nomura, and Y. Miyahara, Polym. J., 9, 371 (1977).

3. F. Eggers, Acustica, 19, 323 (1967).

4. S. B. Dev, S. Sarkar, and R. A. Pethrick, J. Phys. E Sci. Instrum., 6, 139 (1973).

5. A. Labhardt and G. Schwartz, Ber. Bunsenges., 80, 83 (1976).

6. M. A. Cochran, P. B. Jones, A. M. North, and R. A. Pethrick, J. Chem. Soc., Faraday II, 68, 1719 (1972).

7. N. Saito, "Introduction to Polymer Physics" (Japanese), Syokabo, 1967, p 281.

8. S. B. Grigorev, L. A. Kitsenko, and I. G. Mikhailov, Soviet. Phys. Acoust., 22, 281 (1976).

9. Y. Y. Gotlib and A. A. Darinskii, Soviet. Phys. Acoust., 19, 430 (1974).

10. L. A. Davidovich, A. A. Ivanov, S. Makhkamov, L. Pulatova, P. K. Khabibullaev, M. G. Khaliulin, and 
Cylindrical Resonator Technique for Ultrasonic Absorption Measurement

Sh. Sharipov, Soviet. Phys. Acoust., 19, 18 (1973).

11. K. Araki, M. Yabuta, T. Fukuyoshi, and Y. Imamura, Rep. Prog. Polym. Phys. Jpn., 18, 129 (1975)

12. F. W. Wang and B. H. Zimm, J. Phys. Sci., 12, 1619
(1974).

13. P. E. Rouse, J. Chem. Phys., 21, 1272 (1953).

14. B. H. Zimm, J. Chem. Phys., 24, 269 (1956).

15. A. V. Tobolsky and D. B. Dupre, Adv. Polym. Sci., 6, 103 (1969). 